\title{
The Nature of V1464 Aql: A New Ellipsoidal Variable with a $\delta$ Scuti Component
}

\author{
H. A. Dal ${ }^{1,2}$ and E. Sipahi ${ }^{1}$ \\ ${ }^{1}$ Department of Astronomy and Space Sciences, University of Ege, Bornova, 35100 İzmir, Turkey \\ ${ }^{2}$ Corresponding author. Email: ali.dal@ege.edu.tr
}

(Received August 31, 2012; Accepted October 15, 2012; OnLine Publication January 18, 2013)

\begin{abstract}
Taking into account the results obtained from the models and analyses of the BVRI light curves, we discuss the nature of V1464 Aql. The analyses indicated that the mass ratio of the system is $q=0.71 \pm 0.02$, while the inclination of the system (i) is $38^{\circ} .45 \pm 0^{\circ} .22$. Taking the primary component's temperature as $7420 \pm 192 \mathrm{~K}$, we found that the temperature of the secondary is $6232 \pm 161 \mathrm{~K}$. The mass of the primary component was found to be $1.74 \pm 0.05 \mathrm{M}_{\odot}$, while it is $1.23 \pm$ $0.01 \mathrm{M}_{\odot}$ for the secondary. The primary component's radius was found to be $2.10 \pm 0.05 \mathrm{R}_{\odot}$, while it was found as $1.80 \pm 0.01 \mathrm{R}_{\odot}$ for the secondary. Revealing that the system should not exhibit any eclipses, we demonstrated that the main variation with large amplitude should be caused due to the ellipsoidal effect. Indeed, the Fourier analysis also supported the result. For the first time in the literature, we revealed that the primary component is a $\delta$ Scuti star. The period of pulsation was found to be $58.482 \pm 0.002,58.482 \pm 0.001,60.966 \pm 0.002$, and $60.964 \pm 0.003 \mathrm{~min}$ in $B V R I$ bands, respectively. We plotted V1464 Aql in the $\log \left(P_{\text {orb }}\right)-\log \left(P_{\text {pulse }}\right)$ plane. Using more than 160 binaries, whose one or both components are pulsating, we derived a new linear fit in the $\log \left(P_{\text {orb }}\right)-\log \left(P_{\text {pulse }}\right)$ plane for each type of binary. Using the linear fit of each group, we obtained new calibrations between $\log \left(P_{\text {orb }}\right)$ and $\log \left(P_{\text {pulse }}\right)$ for different types of pulsating stars. In addition, a calibration has been obtained for the first time for the pulsating stars with spectral types $O$ and B. V1464 Aql seems to be located near the other ellipsoidal and close binaries. Thus, we listed V1464 Aql as a new candidate for the ellipsoidal variables with a $\delta$ Scuti component.
\end{abstract}

Keywords: binaries: close - binaries: spectroscopic - methods: data analysis - stars: individual (V1464 Aql) - stars: variables: $\delta$ Scuti - techniques: photometric

\section{INTRODUCTION}

The pulsation of a star, as well as it being a component of an eclipsing binary itself, is very important in understanding its evolution. There are several pulsating stars such as Cepheidtype pulsating stars, $\gamma$ Doradus-type pulsating stars, and $\delta$ Scuti-type pulsating stars. All these stars are in the Instability Strip on the main sequence in the Hertzsprung-Russell diagram. An investigation of the frequencies of oscillations, asteroseismology known as stellar seismology, enables us to identify the physical processes behind the pulsating nature and stellar interiors. Pulsating stars play an important role in understanding stellar evolution (Cunha et al. 2007; Aerts, Christensen-Dalsgaard, \& Kurtz 2010). However, in some cases, pulsation features are not enough to solve the entire stellar interior and its nature. In this respect, being a component of a binary can play an important role in solving the problem at hand. In particular, an analysis of the light curve together with radial velocity gives us a large number of physical parameters such as mass, radius, and $\log g$ for components of eclipsing binaries (Wilson \& Devinney 1971; Wilson 1990). Considering both pulsating and eclipsing behaviour, physical natures of stars can be easily identifiable (Lampens 2006; Pigulski 2006). Although there are many pulsating single stars, a few of them are a component in a binary system, especially an eclipsing system. As seen from the literature, the number of pulsating stars is more than 635 , while the incidence of the eclipsing binary component among them is $\sim 5.5 \%$ (Kim et al. 2010).

In this study, we introduce V1464 Aql as a new candidate for a binary with a pulsating component. In the SIMBAD database, V1464 Aql was classified as a spectroscopic binary system from the spectral type of A2V. In the ASAS database (Pojmánski 1997), V1464 Aql's variability type was suggested to be possibly RR Lyr (RRc), or an eclipsing binary, possibly contact (EC), or a semi-detached system 
Table 1. Basic parameters for the observed stars.

\begin{tabular}{lcrc}
\hline \hline Star & $\alpha / \delta(\mathrm{J} 2000)$ & $V(\mathrm{mag})$ & $B-V(\mathrm{mag})$ \\
\hline V1464 Aql & $19^{\mathrm{h}} 50^{\mathrm{m}} 15^{\mathrm{s}} .473 /-08^{\circ} 36^{\prime} 06^{\prime \prime} .26$ & 8.661 & 0.276 \\
GSC 5725 2283 (Comparison) & $19^{\mathrm{h}} 49^{\mathrm{m}} 59^{\mathrm{s}} .290 /-08^{\circ} 35^{\prime} 05^{\prime \prime} .86$ & 10.137 & 1.123 \\
GSC 5725 2387 (Check) & $19^{\mathrm{h}} 50^{\mathrm{m}} 15^{\mathrm{s}} .473 /-08^{\circ} 36^{\prime} 06^{\prime \prime} .26$ & 9.860 & 1.272 \\
\hline
\end{tabular}

Notes. The coordinates were taken from the SIMBAD database, the brightness and colours were taken from the All-Sky Compiled Catalogue of 2.5 million stars (Kharchenko et al. 2009).

(ESD). The system was listed as a contact binary by Duerbeck (1997) and Pribulla \& Rucinski (2006) and a spectroscopic binary by Pourbaix et al. (2004). Duerbeck (1997) listed the period of V1464 Aql as 0.6978, while Rucinski \& Duerbeck (2006) listed it as 0.697822. Detailed spectroscopic observations were made by Rucinski \& Duerbeck (2006). They obtained a single-lined radial velocity with an amplitude of $30.62 \pm 2.35 \mathrm{~km} \mathrm{~s}^{-1}$. They noted that the lines coming from the visible component are largely broadened. The value of $v \sin i$ was found to be $94 \pm 4 \mathrm{~km} \mathrm{~s}^{-1}$. They also claim that the spectral type of the system should be F1-2 rather than early A.

In this study, we investigate the nature of this interesting system. For this purpose, we observed the system photometrically and obtained the multi-band light curves, in which short-term intrinsic light variations are seen for the first time. We analysed the light curves and tested the results with the method based on the Fourier transform described by Morris (1985) and Morris \& Naftilan (1993). Apart from light-curve analyses, we also detected some large-amplitude pulsation with short period. We analysed the pulsating behaviour.

\section{OBSERVATIONS}

Observations were acquired with a thermoelectrically cooled ALTA U+42 2048 $\times 2048$ pixel CCD camera attached to a 40-cm Schmidt-Cassegrains-type MEADE telescope at the Ege University Observatory. Observations were made in BVRI bands during 11 nights on 2011 August 3, 6, 7, 9, 16, and 17, and on 2011 September 4, 8, 15, 19, and 30. V1464 Aql was observed during all the nights in each observation. Some basic parameters of the program stars are listed in Table 1. The names of the stars are listed in the first column, while $\mathbf{J} 2000$ coordinates are listed in the second column. The $V$ magnitudes are in the third column and $B-V$ colours are listed in the last column.

Although program and comparison stars are very close on the sky, using the calibration described by Hardie (1962), the differential atmospheric extinction corrections were applied. Atmospheric extinction coefficients were obtained from observations of the comparison stars on each night. Heliocentric corrections were also applied to the times of the observations.

The mean averages of the standard deviations are 0.023 , $0.011,0.010$, and $0.013 \mathrm{mag}$ for observations acquired in the $B V R I$ bands, respectively. To compute the standard deviations of observations, we used the standard deviations of the reduced differential magnitudes in the sense of comparison minus check stars for each night. No variation was observed in the brightness of comparison stars.

The obtained $B V R I$ data indicated that the system exhibits two main variations in each band. One of them has large amplitude with a longer period, while another has a relatively smaller amplitude with a shorter period. We first investigated the larger variation. For this purpose, we determine the most symmetric and deeper minimum. The light curve obtained in the observations on 2011 September 4 has such a minimum. Using two different methods described by Kwee \& van Woerden (1956) and Winkler (1967), we computed a minimum time. Both methods gave the same minimum time with almost the same error. Then, using the method of the discrete Fourier transform (DFT; Scargle 1982), we analysed its period by the PERIOD04 software (Lenz \& Breger 2005). The results obtained from DFT were tested by two other methods: first, by CLEANest, which is another Fourier method (Foster 1995), and then by the phase dispersion minimization (PDM), which is a statistical method (Stellingwerf 1978). These methods confirmed the results obtained by DFT

$$
\mathrm{JD}(\text { Hel. })=2455809.3467(1)+0^{\mathrm{d}} 697822(3) \times E .
$$

All the ephemerides of the system are given by Equation (1). The analyses reveal that the period of the larger variation is close to those given by Duerbeck (1997) and Rucinski $\&$ Duerbeck (2006). All the observations were phased with using the ephemeris to prepare the light-curve analyses.

\section{LIGHT-CURVE ANALYSES}

\subsection{Eclipsing binary model}

In the literature, V1464 Aql was classified as a contact binary (Duerbeck 1997; Pribulla \& Rucinski 2006). In addition, it was noted in the ASAS database (Pojmánski 1997) that the system could also be an eclipsing binary, especially a contact binary. Considering this knowledge, we tried to model the light curves with the Wilson-Devinney code (Wilson \& Devinney 1971; Wilson 1990). For this purpose, we analysed the light curves obtained in the $B V R I$ bands together with the available single-lined radial velocity curve simultaneously, using the PHOEBE V.0.31 a software (Prša \& Zwitter 2005), which is used in the version 2003 of the Wilson-Devinney code (Wilson \& Devinney 1971; Wilson 1990). We tried to analyse the light curves with several different modes, such as 
the 'overcontact binary not in thermal contact' and 'double contact binary' modes. Initial analyses demonstrated that an astrophysically acceptable result can be obtained if the analysis is carried out in the 'double contact binary' mode, while no acceptable results could be obtained in the other modes.

In the literature, temperature is given in a range from $6843 \mathrm{~K}$ (Ammons et al. 2006) to $8970 \mathrm{~K}$ (Wright et al. 2003) for V1464 Aql. We took JHK brightness of the system $(J=7.963 \pm 0.027 \mathrm{mag}, H=7.830 \pm 0.027$ mag, $K=7.788 \pm 0.020 \mathrm{mag}$ ) from the NOMAD catalogue (Zacharias et al. 2005). Using these brightnesses, we derived de-reddened colours as $(J-H)_{0}=0.110 \pm 0.038 \mathrm{mag}$ and $(H-K)_{0}=0.030 \pm 0.034 \mathrm{mag}$ for the system. Using the calibrations given by Tokunaga (2000), we derived the temperature of the primary component as $7420 \pm 192 \mathrm{~K}$ depending on these de-reddened colours. Taking into account the standard deviation of the brightness in $J H K$ bands, we computed the standard deviation of the temperature as $192 \mathrm{~K}$. Thus, the temperature was found to be $7420 \pm 192 \mathrm{~K}$. Thus, the temperature of the primary component was fixed to $7420 \pm 192 \mathrm{~K}$ in the analyses, and that of the secondary was taken as a free parameter. Considering the spectral type corresponding to this temperature, the albedos $\left(A_{1}\right.$ and $\left.A_{2}\right)$ and the gravity-darkening coefficients $\left(g_{1}\right.$ and $\left.g_{2}\right)$ of the components were adopted for the stars with the convective envelopes (Lucy 1967; Rucinski 1969). The nonlinear limb-darkening coefficients $\left(x_{1}\right.$ and $\left.x_{2}\right)$ of the components were taken from van Hamme (1993). In the analyses, their dimensionless potentials $\left(\Omega_{1}\right.$ and $\left.\Omega_{2}\right)$, the fractional luminosity $\left(L_{1}\right)$ of the primary component, the inclination $(i)$ of the system, the mass ratio of the system $(q)$, and the semi-major axis $(a)$ were taken as the adjustable free parameters.

Because it is single lined, the available radial velocity curve does not give any mass ratio for the system. Therefore, considering general weighted sum of squared residuals $\left(\Sigma \mathrm{res}^{2}\right)$, we tried to find the mass ratio of the system. In analyses, $\Sigma$ res $^{2}$ values indicated that the photometric mass ratio of the components is $q=0.71 \pm 0.02$. According to this result, we assume that the mass ratio of the system is $q=0.71 \pm 0.02$. The temperature of the secondary component was found to be $6232 \mathrm{~K}$ and its error as $27 \mathrm{~K}$. However, the error seems to be unreal in the statistical sense. Taking into account the standard deviation of the brightness in JHK bands, we have computed the standard deviation of the temperature as $161 \mathrm{~K}$. Finally, the temperature was found to be $6232 \pm 161 \mathrm{~K}$ for the secondary component. All the parameters derived from the analyses are listed in Table 2, while the synthetic light curves are shown in Figure 1. In addition, the radial velocity obtained by Rucinski \& Duerbeck (2006) and the synthetic curve are shown in Figure 2. Finally, we also derived the 3D model of Roche geometry and the geometric configurations at four special phases $0.00,0.25$, 0.50 , and 0.75 for the system, using the parameters obtained from the light-curve analysis. This 3D model and geometric configurations are shown in Figure 3.
Table 2. Parameters of the components obtained from the light-curve analysis.

\begin{tabular}{lc}
\hline \hline Parameter & Value \\
\hline Orbital period $(P)$ & $0.697822 \mathrm{~d}$ \\
Mass ratio $\left(M_{2} / M_{1}=q\right)$ & $0.71 \pm 0.02$ \\
Inclination $(i)$ & $38^{\circ} .45 \pm 0^{\circ} .22$ \\
Temperature $\left(T_{1}\right)$ & $7420 \pm 192^{a} \mathrm{~K}$ \\
Temperature $\left(T_{2}\right)$ & $6232 \pm 161 \mathrm{~K}$ \\
Dimensionless potential $\left(\Omega_{1}\right)$ & $3.2592 \pm 0.0006$ \\
Dimensionless potential $\left(\Omega_{2}\right)$ & $3.2582 \pm 0.0006$ \\
Fractional luminosity $\left(L_{1} / L_{T}, B\right)$ & $0.673 \pm 0.049$ \\
Fractional luminosity $\left(L_{1} / L_{T}, V\right)$ & $0.646 \pm 0.042$ \\
Fractional luminosity $\left(L_{1} / L_{T}, R\right)$ & $0.623 \pm 0.037$ \\
Fractional luminosity $\left(L_{1} / L_{T}, I\right)$ & $0.605 \pm 0.031$ \\
Gravity-darkening coefficients $\left(g_{1}, g_{2}\right)$ & $1.00,0.32$ \\
Albedo $\left(A_{1}, A_{2}\right)$ & $1.00,0.50$ \\
Limb-darkening coefficients $\left(x_{1, \text { bol }}, x_{2, \text { bol }}\right)$ & $0.657,0.657$ \\
Limb-darkening coefficients $\left(x_{1, B}, x_{2, B}\right)$ & $0.808,0.809$ \\
Limb-darkening coefficients $\left(x_{1, V}, x_{2, V}\right)$ & $0.703,0.704$ \\
Limb-darkening coefficients $\left(x_{1, R}, x_{2, R}\right)$ & $0.592,0.592$ \\
Limb-darkening coefficients $\left(x_{1, I}, x_{2, I}\right)$ & $0.489,0.489$ \\
Fractional radius $\left(\left\langle r_{1}\right\rangle\right)$ & $0.441 \pm 0.002$ \\
Fractional radius $\left(\left\langle r_{2}\right\rangle\right)$ & $0.378 \pm 0.002$ \\
Absolute luminosity $\left(L_{1}\right)$ & $11.97 \pm 0.31 \mathrm{~L}_{\odot}$ \\
Absolute luminosity $\left(L_{2}\right)$ & $4.41 \pm 0.02 \mathrm{~L}_{\odot}$ \\
Semi-amplitude of radial velocity $\left(\mathrm{km} \mathrm{s} \mathrm{s}^{-1}\right)$ & $30.8132^{+0.3705}-0.3615$ \\
Systemic velocity $\left(\mathrm{km} \mathrm{s} \mathrm{s}^{-1}\right)$ & $16.2560^{+0.2434}-0.2469$ \\
\hline \hline
\end{tabular}

${ }^{a}$ The error is computed depending on the standard deviations of dereddened colours.

Although no double-lined radial velocity curve is available, we tried to estimate absolute parameters of the components. According to Tokunaga (2000), the mass of the primary component must be $1.74 \pm 0.05 \mathrm{M}_{\odot}$, corresponding to its surface temperature. Considering possible mass ratio of the system, the mass of the secondary component was found to be $1.23 \pm 0.01 \mathrm{M}_{\odot}$.

Using Kepler's third law, we calculated the semi-major axis as $4.76 \pm 0.03 \mathrm{R}_{\odot}$. Considering this estimated semimajor axis, the radius of the primary component was computed as $2.10 \pm 0.05 \mathrm{R}_{\odot}$, while that for the secondary component it was $1.80 \pm 0.01 \mathrm{R}_{\odot}$. Using the estimated radii and the obtained temperatures of the components, the luminosity of the primary component was estimated to be $11.97 \pm 0.31 \mathrm{~L}_{\odot}$ and that of the secondary component it was $4.41 \pm 0.02 \mathrm{~L}_{\odot}$. We have plotted the distribution of radii versus masses and luminosity versus temperature for some stars in Figure 4. The lines represent the ZAMS: Zero-age main sequence theoretical model developed for the stars with $Z=0.02$ by Girardi et al. (2000), while dashed lines represent the TAMS: Terminal-age main sequence theoretical model. According to the results, the absolute parameters are generally acceptable in the astrophysical sense.

\subsection{Elipsoidal binary model}

As seen from the Roche geometry shown in Figure 3 and results of the light curve analysis, the system does not exhibit 


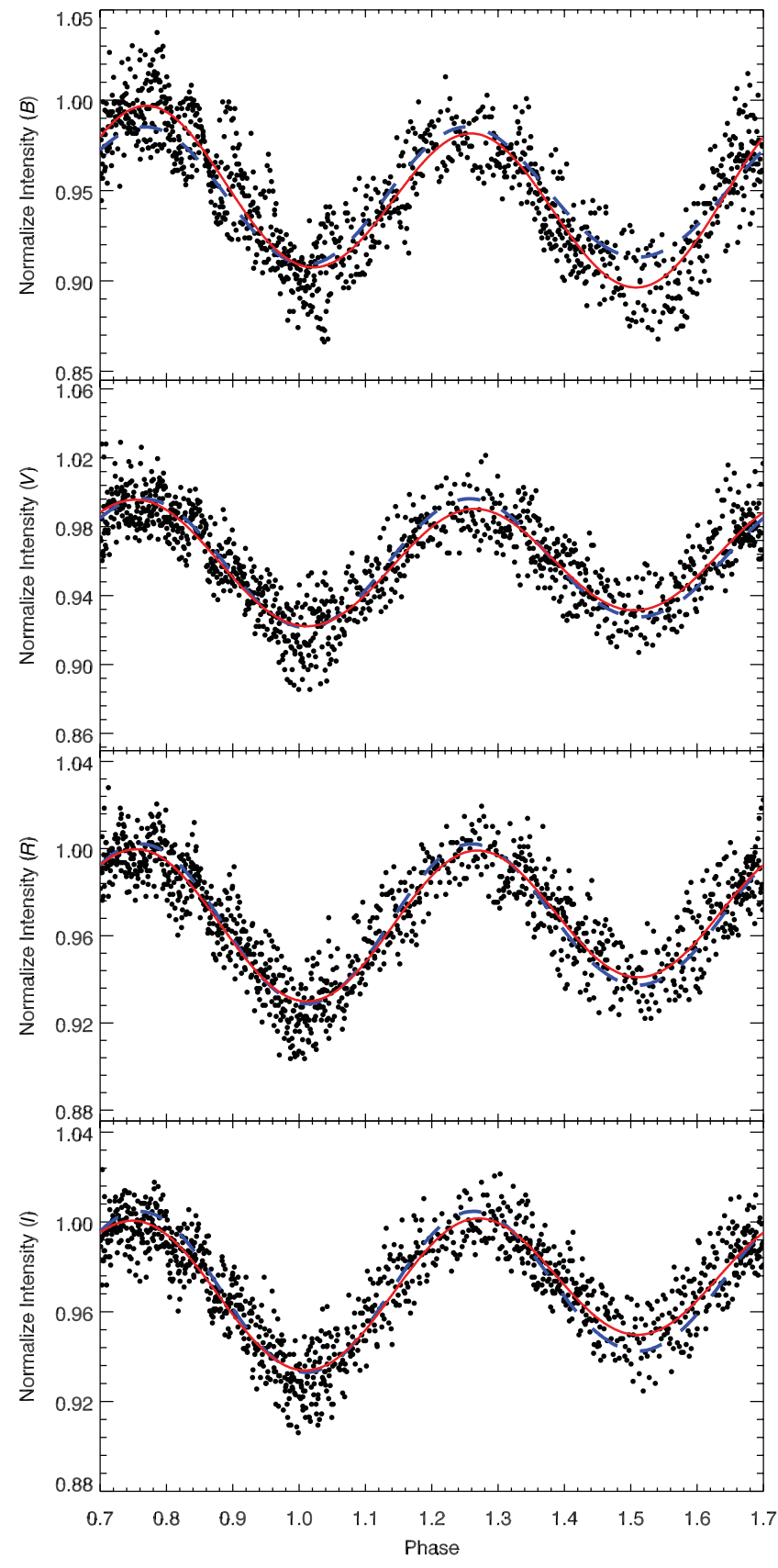

Figure 1. Observed $B V R I$ light curves and synthetic curves obtained from the light-curve analysis and Fourier models. The filled circles represent the observations, while the dashed (blue) lines represent the synthetic curves obtained from the light-curve analysis and the (red) line represents the derived Fourier fits.

any eclipses. However, the shape of the components should cause some light variation, known as the ellipsoidal variation. Ellipsoidal variable stars are non-eclipsing binary stars and especially close binaries (Morris 1985; Beech 1985). In the case of the ellipsoidal variables, the inclination angle $(i)$ of the binary is so small that the system does not exhibit any eclipses. The main variation is due to non-spherical shapes of the components. According to Morris (1985), Beech (1985), and Morris \& Naftilan (1993), if the main variation occurs due to the ellipsoidal effect, the light curves of the ellipsoidal variables can be modelled by the Fourier analysis given by Equation (2), and one will also expect that the $\cos (2 \theta)$ term must be dominant among all other terms in the results of the Fourier analysis. This is the base of the modern methods used in recent studies, such as Faigler \& Mazeh (2011) and Faigler et al. (2012):

$$
L(\theta)=A_{0}+\sum_{\mathrm{i}=1}^{N} A_{i} \cos (i \theta)+\sum_{\mathrm{i}=1}^{N} B_{i} \sin (i \theta) .
$$

All the BVRI light curves were modelled with Fourier series. The derived Fourier models are shown in Figure 1 and Fourier coefficients are listed in Table 3 . The $A_{i}$ coefficients listed in the table are the coefficients of the $\cos (i \theta)$ terms, while $B_{i}$ parameters are the coefficients of the $\sin (i \theta)$ terms given in Equation (2). In fact, the most dominant one is the $\cos (2 \theta)$ term for each of the BVRI bands. Thus, it is obvious that the main effect seen in the light variations is the ellipticity effect. However, if the most dominant one were the $\cos (\theta)$ term, we would consider the other effects such as magnetic activity occurring on the surface of cool stars. There are many systems that are similar to V1464 Aql, such as 75 Pegasi and 42 Persei (Martin, Hube, \& Lyder 1990; Martin, Hube, \& Brown 1991), including several others (Faigler \& Mazeh 2011; Faigler et al. 2012).

\section{THE PULSATION}

Apart from the main variation, there is a shorter period oscillation. To understand the shorter period oscillation, first we obtained all the pre-whitened light curves. To obtain the pre-whitened data, we extracted synthetic light curves from all the observations in each band. In the second step, all the pre-whitened data were analysed with both the DFT (Scargle 1982) and PDM methods (Stellingwerf 1978).

The periods found from the pre-whitened data are listed in Table 4. The normalised power spectra, which exhibit the quality of the period analysis, are shown in Figure 5. Using the light elements given in Equation (1) and the parameters found from the pre-whitened data, all the nightly light variations were modelled for each band. The modelled light variations are shown in Figure 6.

Soydugan et al. (2006) listed some eclipsing binaries whose primary and/or secondary components are pulsating. Using the physical parameters found in this study, the location of V1464 Aql's primary component is shown in Figure 7. In this figure, following Soydugan et al. (2006), the ZAMS (broad line) and TAMS (dashed line) were taken from Girardi et al. (2000). The borders of the Instability Strip on the main sequence were computed from Rolland et al. (2002). We show that V1464 Aql's primary component is located among the eclipsing binaries, whose one or two components are in the Instability Strip on the main sequence. The primary component of V1464 Aql is at least a pulsating variable for the case. In the literature, there are also four ellipsoidal 


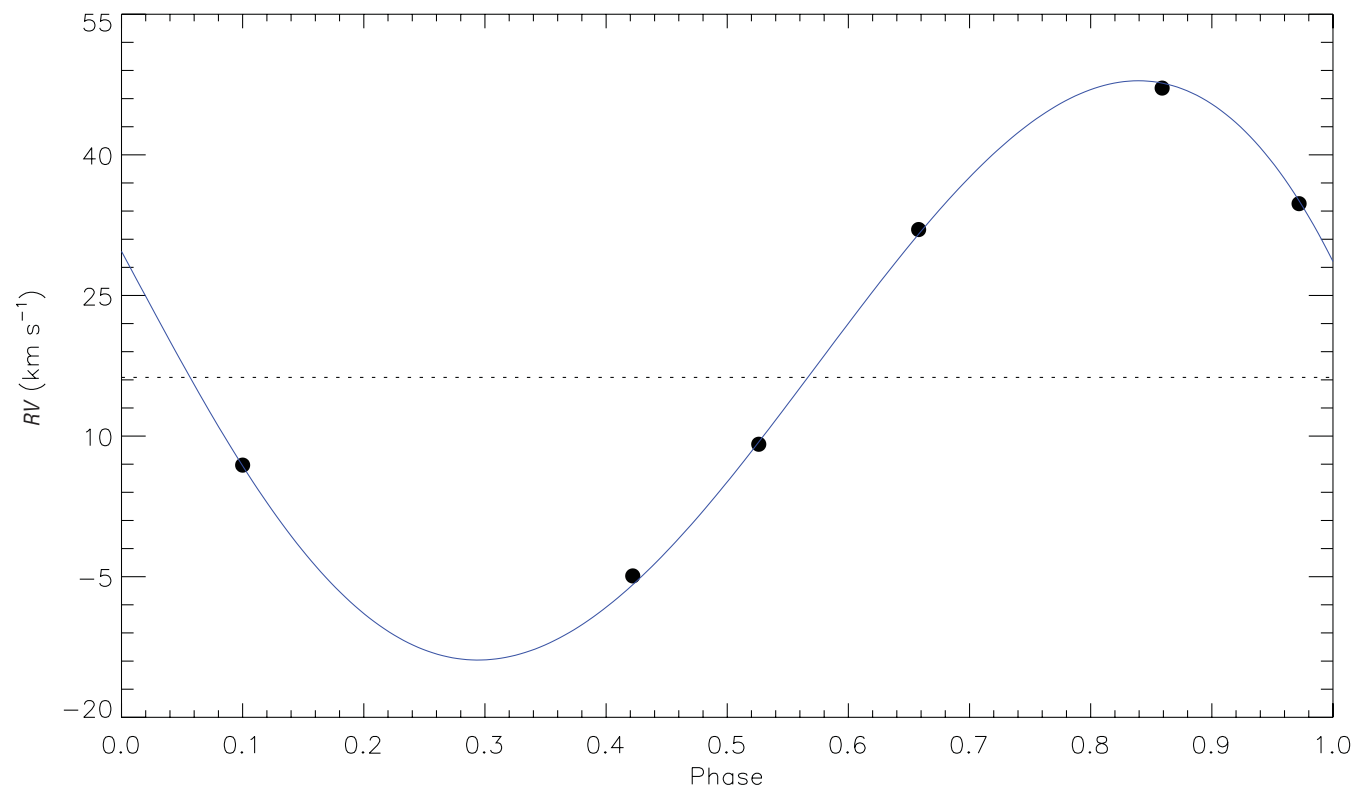

Figure 2. Radial velocity (filled circles) obtained by Rucinski \& Duerbeck (2006) and the synthetic curve (blue line) derived by using parameters found from the light-curve analysis. The dotted line represents the center-of-mass velocity.

Table 3. Coefficients derived from the Fourier model.

\begin{tabular}{lcrccc}
\hline \hline Filter & $A_{0}$ & \multicolumn{1}{c}{$A_{1}$} & \multicolumn{1}{c}{$A_{2}$} & $B_{1}$ & \multicolumn{1}{c}{$B_{2}$} \\
\hline$B$ & $-2.0271 \pm 0.0006$ & $-0.0075 \pm 0.0008$ & $0.0492 \pm 0.0008$ & $0.0078 \pm 0.0008$ & $0.0091 \pm 0.0008$ \\
$V$ & $-1.1129 \pm 0.0005$ & $0.0052 \pm 0.0006$ & $0.0372 \pm 0.0007$ & $0.0032 \pm 0.0006$ & $0.0041 \pm 0.0006$ \\
$R$ & $-0.6554 \pm 0.0004$ & $0.0064 \pm 0.0006$ & $0.0355 \pm 0.0006$ & $0.0008 \pm 0.0006$ & $0.0052 \pm 0.0005$ \\
$I$ & $-0.2349 \pm 0.0004$ & $0.0092 \pm 0.0005$ & $0.0327 \pm 0.0005$ & $0.0000 \pm 0.0005$ & $0.0041 \pm 0.0005$ \\
\hline \hline
\end{tabular}

variables with a $\delta$ Scuti component. As listed in Table 5, these are XX Pyx (Aerts et al. 2002), HD 173977 (Chapellier et al. 2004), HD 207651 (Henry, Fekel, \& Henry 2004), and HD 149420 (Fekel \& Henry 2006). In this case, V1464 Aql is the fifth ellipsoidal variable binary with a $\delta$ Scuti component.

As shown by Turner (2011) and Liakos et al. (2012), close binaries with a pulsating component exhibit some calibrations in the $\log \left(P_{\text {orb }}\right)-\log \left(P_{\text {pulse }}\right)$ plane. For this purpose, we took the data of all listed close binaries, with A or $\mathrm{F}$ spectral type components, from Turner (2011) and Liakos et al. (2012). We also took the data of listed binaries, with components generally from $\mathrm{O}$ or B spectral types, from Aerts $\&$ Harmanec (2004). In addition, we plotted all the long secondary period variables (LSPVs) listed by Kiss et al. (1999) and Olivier \& Wood (2003) in the same plane. In the case of binaries with $\mathrm{A}$ and $\mathrm{F}$ spectral type components, the pulsating components are $\delta$ Scuti stars, while the other components are pulsating stars except $\delta$ Scuti star for binaries, with spectral type component $\mathrm{O}$ or B. Although LSPVs have been a subject of debate in the literature, a relation exists between their periods (Kiss et al. 1999; Olivier \& Wood 2003). In Figure 8, we plotted all the binaries in the $\log \left(P_{\text {orb }}\right)-\log \left(P_{\text {pulse }}\right)$ plane. All the components plotted in this figure are pulsating stars.
Moreover, we plotted the pulsating components of all known ellipsoidal variables, whose one component is a $\delta$ Scuti star:

$$
\begin{aligned}
& \log \left(P_{\text {pulse }}\right)=-0.040(0.070) \times \log \left(P_{\text {orb }}\right)-0.337(0.025), \\
& \log \left(P_{\text {pulse }}\right)=0.593(0.041) \times \log \left(P_{\text {orb }}\right)-1.545(0.021), \\
& \log \left(P_{\text {pulse }}\right)=1.083(0.024) \times \log \left(P_{\text {orb }}\right)-1.449(0.059) .
\end{aligned}
$$

Using the GraphPad Prism V5.02 software (Motulsky 2007), we re-modelled the distribution of each group with the linear fit. Here, the standard deviations of each coefficient and each constant are given in parentheses. The derived linear fits are also plotted in Figure 8. To test whether the derived linear fits are statistically acceptable, we computed the probability value (hereafter $\mathrm{p}$ value). The threshold level of significance ( $\alpha$ value) was taken to be 0.005 for the $\mathrm{p}$ value, which allowed us to test whether the $\mathrm{p}$ values are statistically acceptable or not (Wall \& Jenkins 2003). The derived linear fit is given in Equation (3) for the binaries with spectral type components $\mathrm{O}$ and $\mathrm{B}$. The $\mathrm{p}$ value was found to be $<0.0027$. The linear fit of the $\mathrm{A}-\mathrm{F}$ binaries is given in 
3D model of Roche lobes:
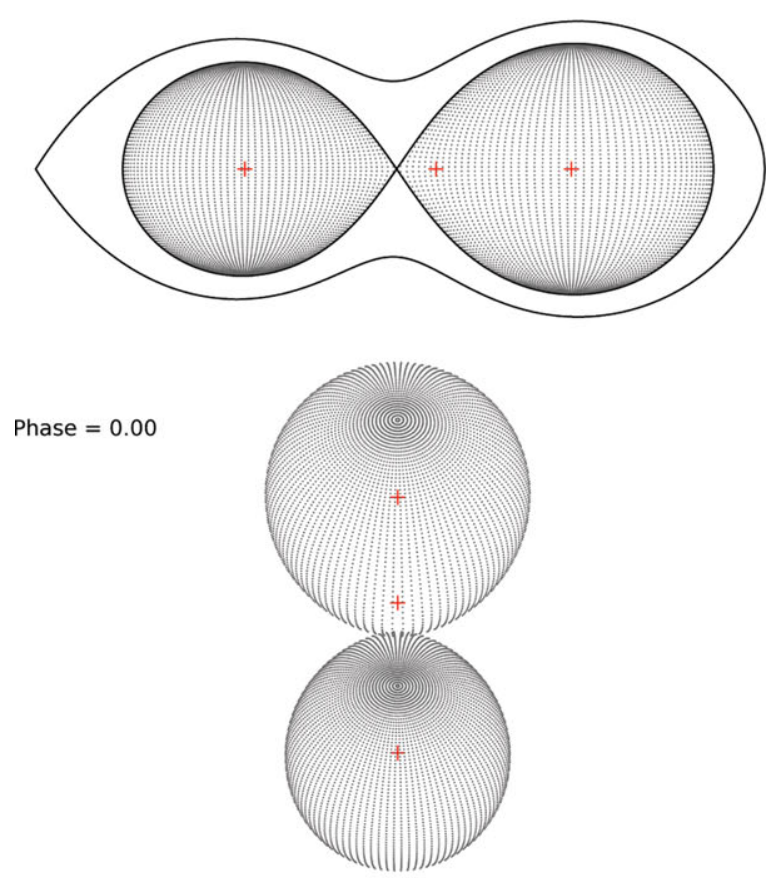

Phase $=0.25$

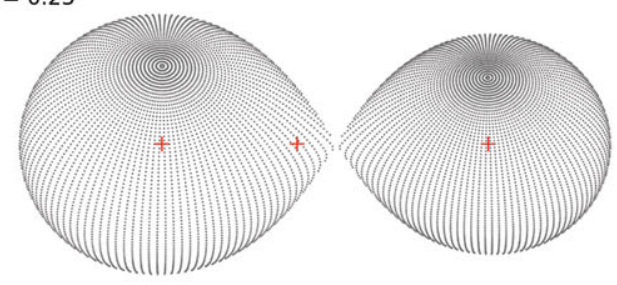

Phase $=0.50$

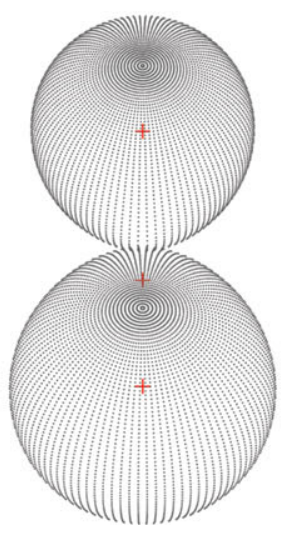

Figure 3. 3D model of Roche geometry and geometric configurations at four special phases $0.00,0.25,0.50$, and 0.75, illustrated for V1464 Aql, using parameters obtained from the light-curve analysis.

Equation (4), while that for the ellipsoidal variables is given in Equation (5). The computed $p$ values are $<0.0001$ for both groups. Considering the $\alpha$ value, the derived linear fits are statistically acceptable.

As Figure 8 shows in the plane of the $\log \left(P_{\text {orb }}\right)-\log \left(P_{\text {pulse }}\right)$ distribution, V1464 Aql is located near the close binaries and ellipsoidal variables with spectral types A and F.
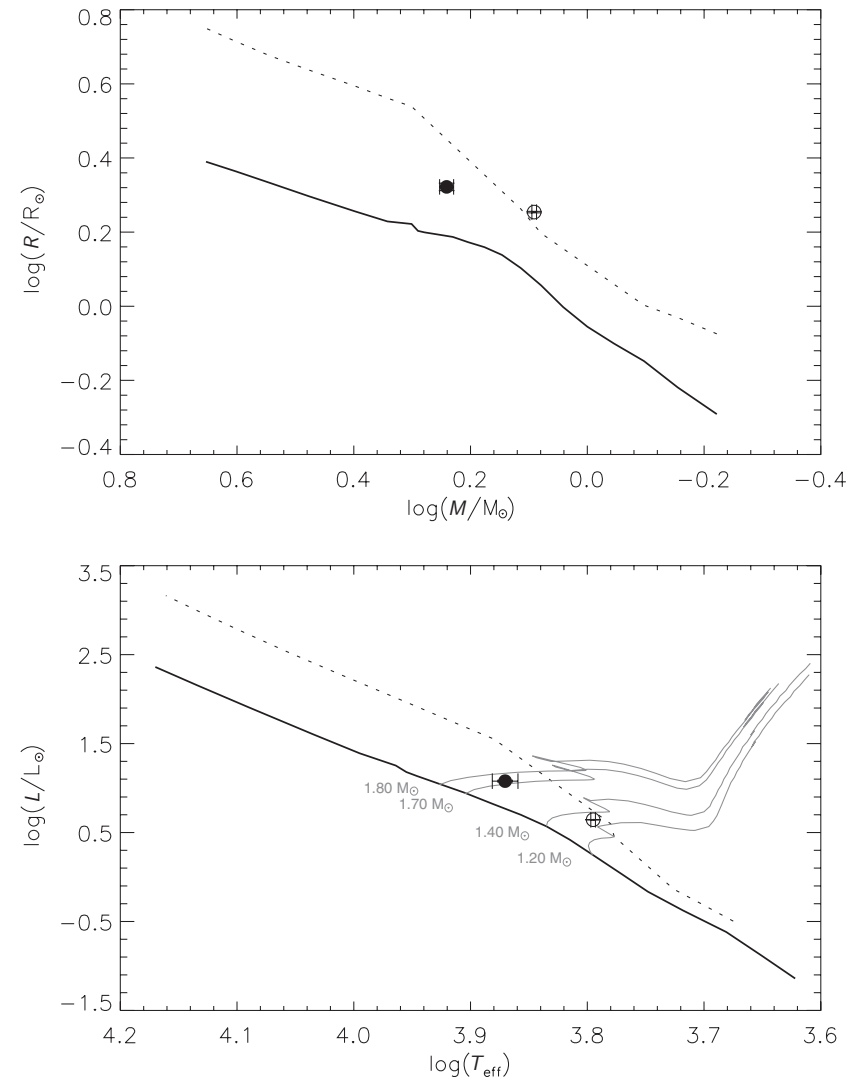

Figure 4. Locations of the components of V1464 Aql in the mass-radius (upper panel) and temperature-luminosity (bottom panel) distributions. The lines represent the ZAMS theoretical model developed by Girardi et al. (2000), while the dashed lines represent the theoretical TAMS model. The grey lines represent the theoretical evolutional tracks. The filled circles represent the primary component, while the open circles represent the secondary component.

Table 4. Pulsation period found from period analyses in each filter.

\begin{tabular}{lcc}
\hline \hline Filter & Period (min) & Amplitude (mag) \\
\hline$B$ & $58.482 \pm 0.002$ & $0.030 \pm 0.002$ \\
$V$ & $58.482 \pm 0.001$ & $0.024 \pm 0.003$ \\
$R$ & $60.966 \pm 0.002$ & $0.017 \pm 0.006$ \\
$I$ & $60.964 \pm 0.003$ & $0.011 \pm 0.006$ \\
\hline \hline
\end{tabular}

\section{RESULTS AND DISCUSSION}

In the literature, there are several stars or systems which exhibit combinations of a few different variations. Some of them are eclipsing binaries with a pulsating component, and some semi-regular variables with unknown short-term variations or some pulsating ellipsoidal variables (Derekas et al. 2006; Nie, Zhang, \& Jiang 2010; Faigler \& Mazeh 2011; Faigler et al. 2012).

In several studies, V1464 Aql was classified as a spectroscopic binary system, RR Lyr (RRc), an eclipsing binary, possibly contact (EC) or semi-detached system (ESD) (Duerbeck 1997; Pourbaix et al. 2004; Pribulla \& Rucinski 2006; 

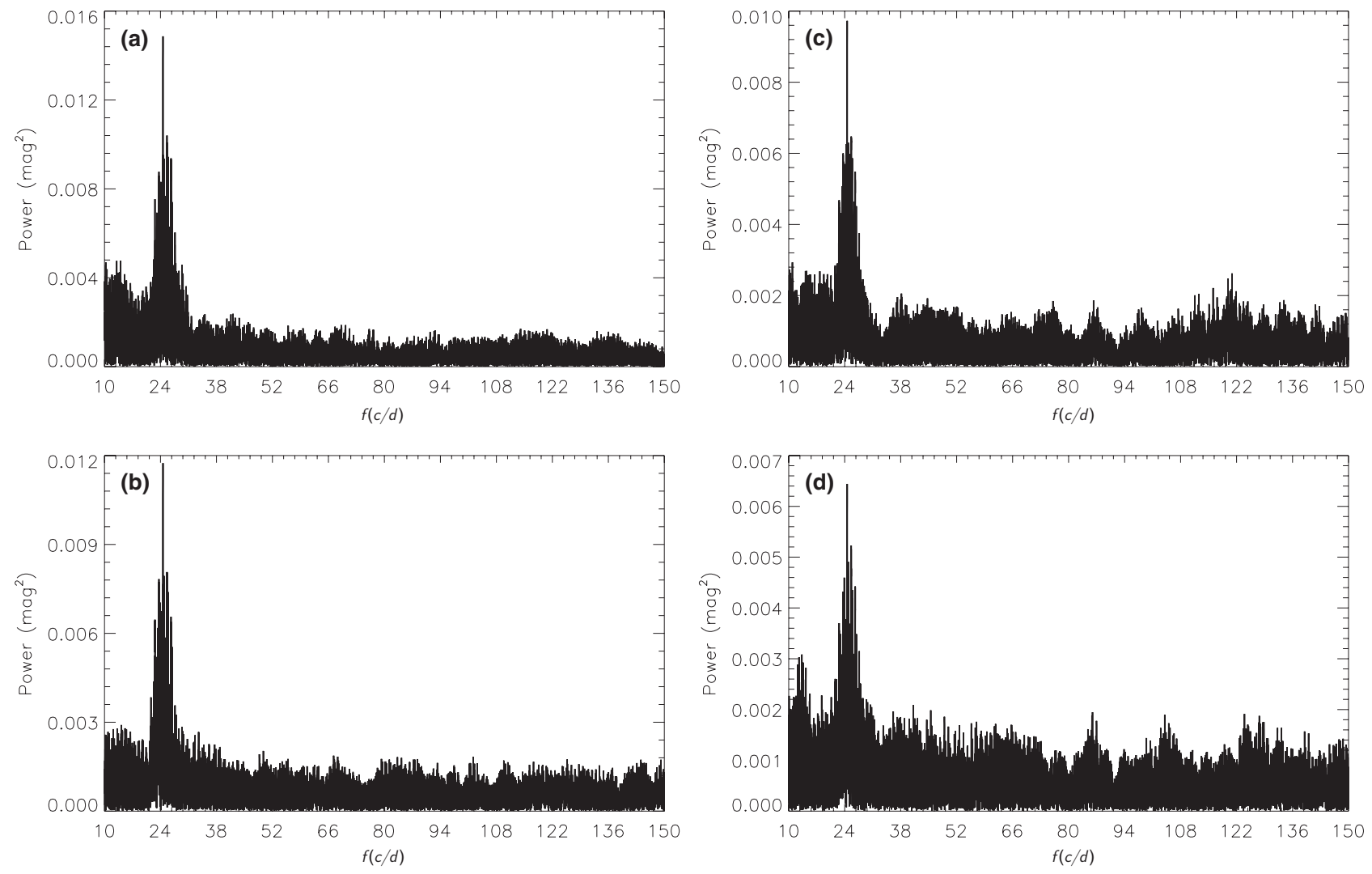

Figure 5. V1464 Aql's normalised power spectra obtained from period analyses of the pre-whitened data with the DFT method in each band. The power spectra obtained from $B V R I$ bands are given in panels (a-d) respectively.

Table 5. Known ellipsoidal binaries having a pulsating component in the literature.

\begin{tabular}{lccccccccc}
\hline \hline Stars & $\begin{array}{c}\text { Spectral } \\
\text { type }^{a}\end{array}$ & $\begin{array}{c}V \\
(\mathrm{mag})\end{array}$ & $\begin{array}{c}P_{\text {orb }} \\
(\mathrm{d})\end{array}$ & $\begin{array}{c}P_{\text {pulse }} \\
(\mathrm{d})\end{array}$ & $\begin{array}{c}M_{1} \\
\left(\mathrm{M}_{\odot}\right)\end{array}$ & $\begin{array}{c}M_{2} \\
\left(\mathrm{M}_{\odot}\right)\end{array}$ & $\begin{array}{c}R_{1} \\
\left(\mathrm{R}_{\odot}\right)\end{array}$ & $\begin{array}{c}R_{2} \\
\left(\mathrm{R}_{\odot}\right)\end{array}$ & $\begin{array}{l}\text { Ref. } \\
\text { XX Pyx }\end{array}$ \\
A4 & 11.490 & 1.150000 & 0.02624 & 1.85 & - & 1.95 & - & $b$ \\
HD207651 & A5 & 7.210 & 1.470800 & 0.73540 & - & - & - & - & $c$ \\
HD173977 & F2 & 8.070 & 1.800745 & 0.06889 & 1.87 & 1.30 & 2.87 & 1.42 & $d$ \\
HD149420 & A8 & 6.874 & 3.394306 & 0.07608 & - & - & - & - & $e$ \\
\hline \hline
\end{tabular}

${ }^{a}$ Taken from the SIMBAD database.

${ }^{b}$ Aerts et al. (2002).

${ }^{c}$ Henry et al. (2004).

${ }^{d}$ Chapellier et al. (2004).

${ }^{e}$ Fekel \& Henry (2006).

Rucinski \& Duerbeck 2006). We analysed the BVRI light curves. As shown in Table 2, the inclination of the system $(i)$ is found to be $38^{\circ} .45 \pm 0^{\circ} .22$. In the case of this (i) value, the system does not exhibit any eclipses. As shown in Figure 3, the 3D geometric configurations derived at four special phases for the system also demonstrated the noneclipsing cases. However, these configurations indicate that the system is on the edge of eclipsing. Although the system may exhibit grazing eclipses, these eclipses are not seen in other small variability. As estimated in the literature, the 3D model of Roche geometry reveals that the components of the system are close to filling their Roche lobes. According to these results, V1464 Aql should be a candidate of the contact binary system, but non-eclipsing due to the inclination of the system $(i)$. The Fourier analysis also supported this result. The coefficients of the $\cos (2 \theta)$ term $\left(A_{2}\right)$ were found to be $0.0492 \pm 0.0008,0.0372 \pm 0.0007,0.0355 \pm 0.0006$, and $0.0327 \pm 0.0005$ for the BVRI bands, respectively. According to these values, the main effect, known as the ellipsoidal effect, in the light variation of the system comes from the shape of the components.

According to the calibration described by Tokunaga (2000), the mass of the primary component should be $1.74 \pm 0.05 \mathrm{M}_{\odot}$ corresponding to its surface temperature. Under this assumption, the light-curve analysis reveals that the temperature of the secondary component was found to 

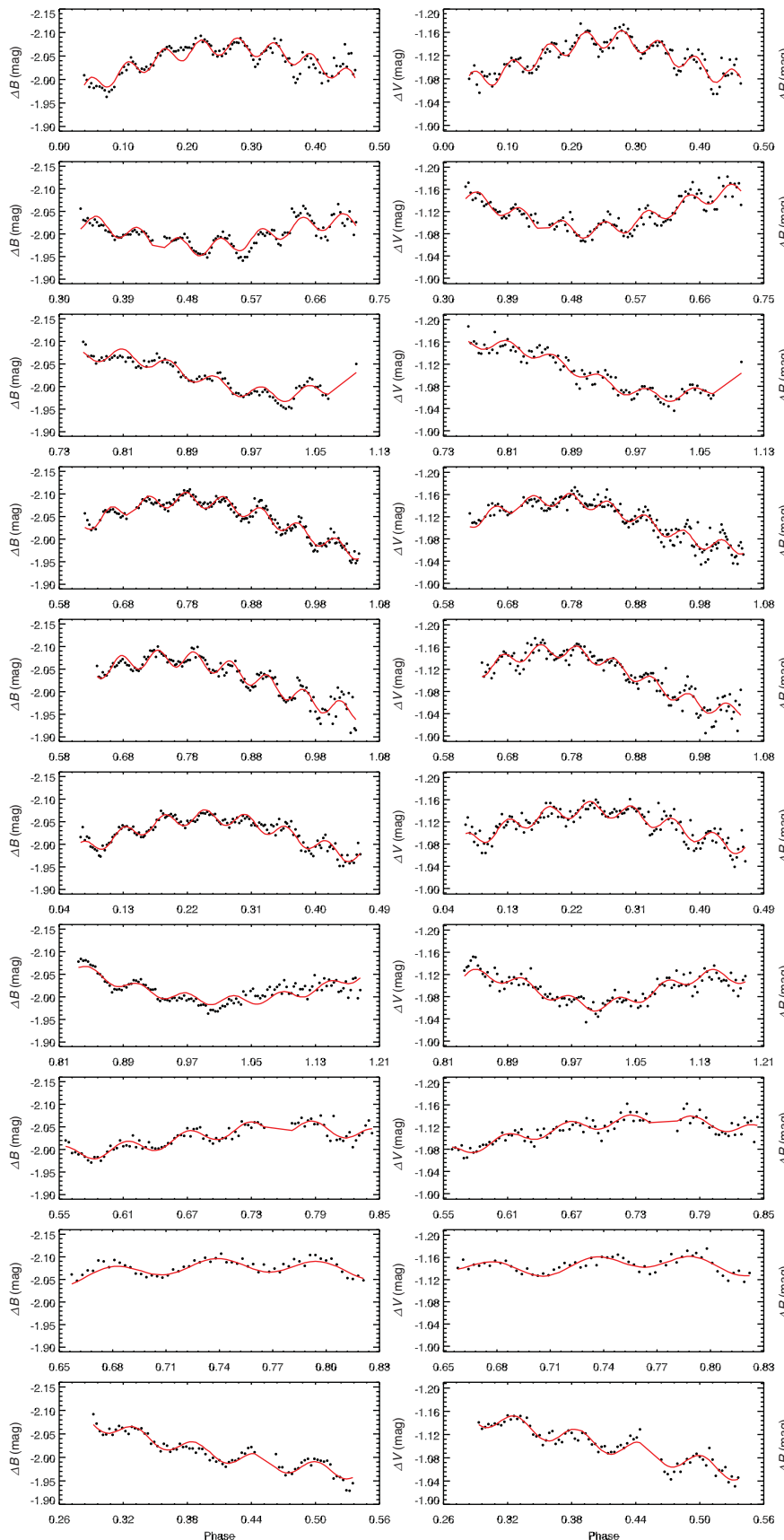

Figure 6. All the models of nightly light variations.

be $6232 \pm 161 \mathrm{~K}$. The light-curve analysis indicates that the mass ratio of the system should be $q=0.71 \pm 0.02$. Note that the standard deviation of the primary component temperature and absolute parameters were computed depending on the standard deviations given for the colours in the NOMAD catalogue (Zacharias et al. 2005). However, the error in temperature was found from the light-curve analysis for the secondary component. Considering the standard deviations of other parameters, it seems slightly lower than expected. The locations of the components plotted in Figure 4 demonstrated that the results of the analysis are acceptable
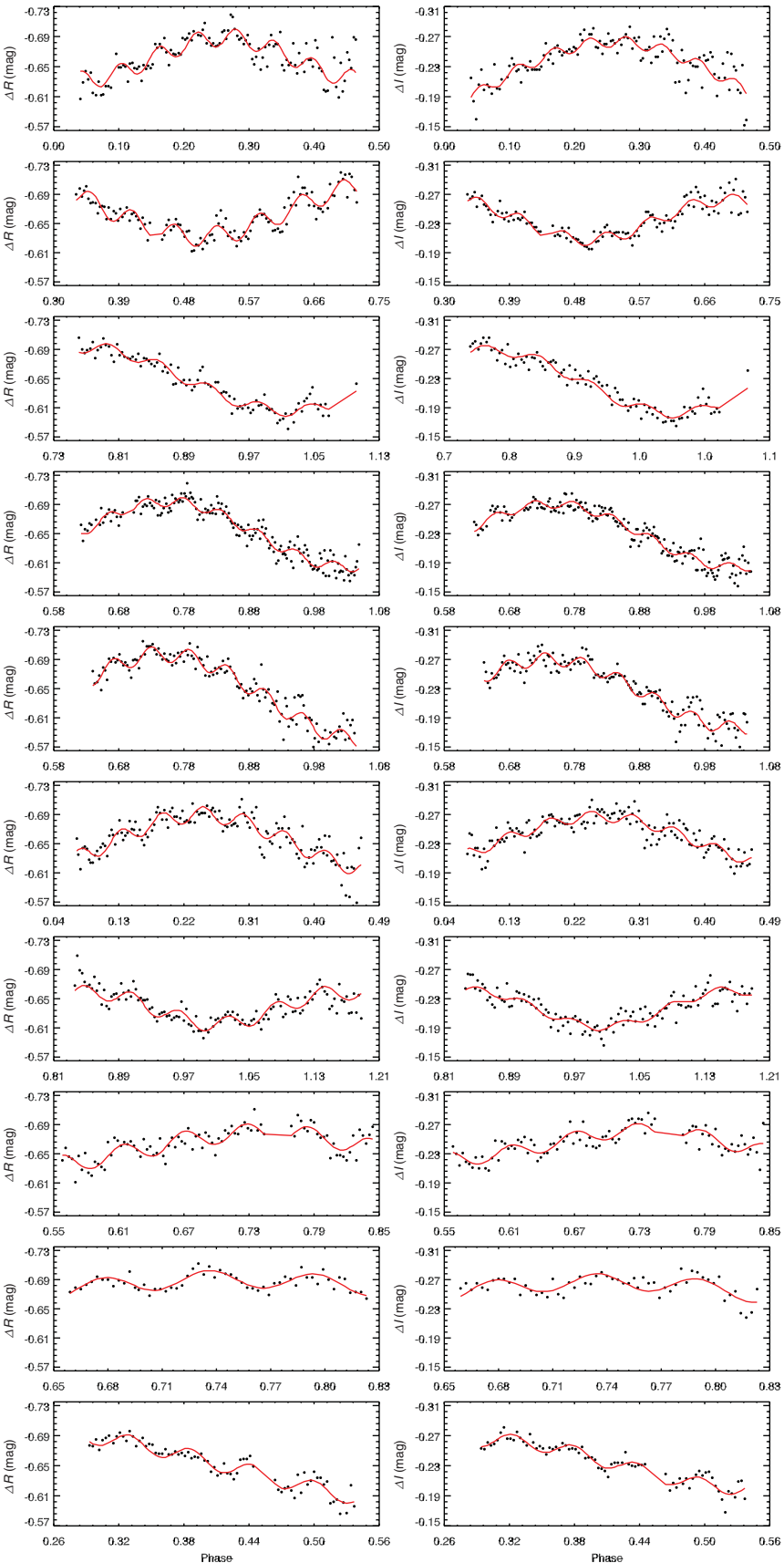

in the astrophysical sense. On the contrary, the secondary component is seen closer to the TAMS than the primary in Figure 4. The stars seem not to be coeval to each other. However, it is well known that there are several binaries such as YY CrB (Essam et al. 2010), BS Cas (Yang, Wei, \& He 2008), VZ Tri (Yang 2010). All these samples exhibit the same behaviour. Their common properties are that all of them are contact binaries with a large period variation due to the large mass transfer. Indeed, if V1464 Aql is an analogue of these systems, this makes V1464 Aql very important for future studies. 


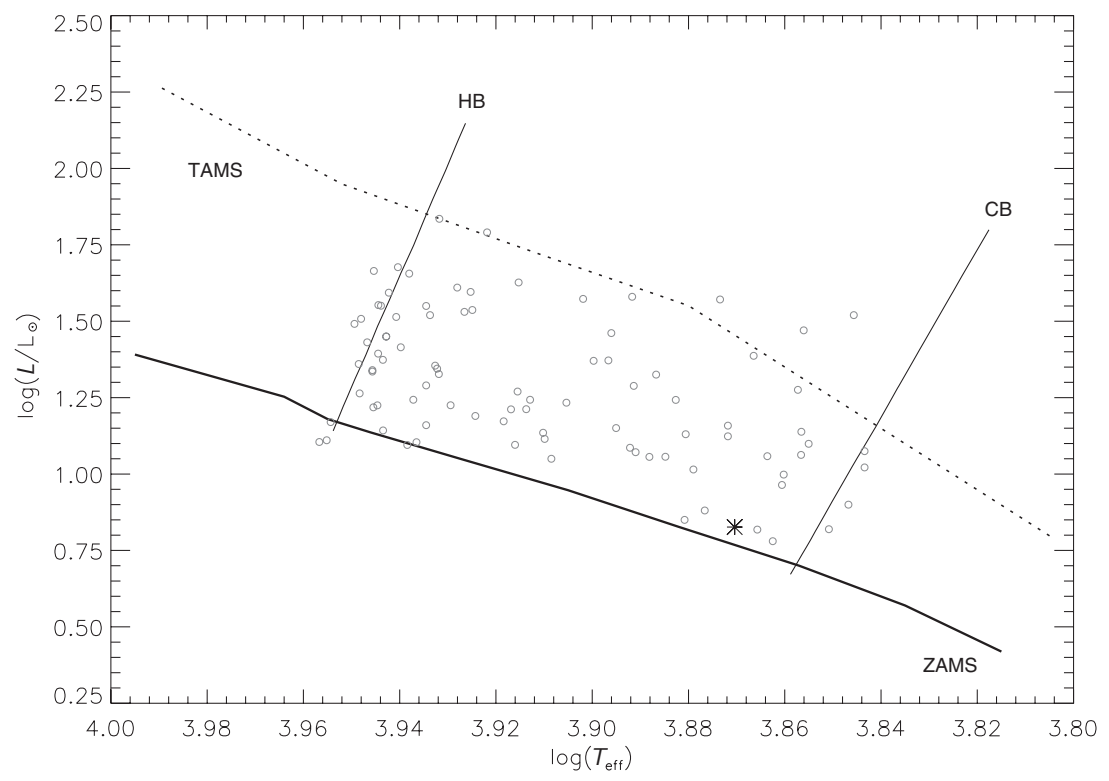

Figure 7. Location of V1464 Aql's primary component in the Instability Strip on the main sequence. The asterisk represents the primary component of V1464 Aql, while the dim circles represent some semi- and undetached binaries taken from Soydugan et al. (2006) and references therein. ZAMS and TAMS were taken from Girardi et al. (2000), while the borders of the Instability Strip were computed from Rolland et al. (2002).

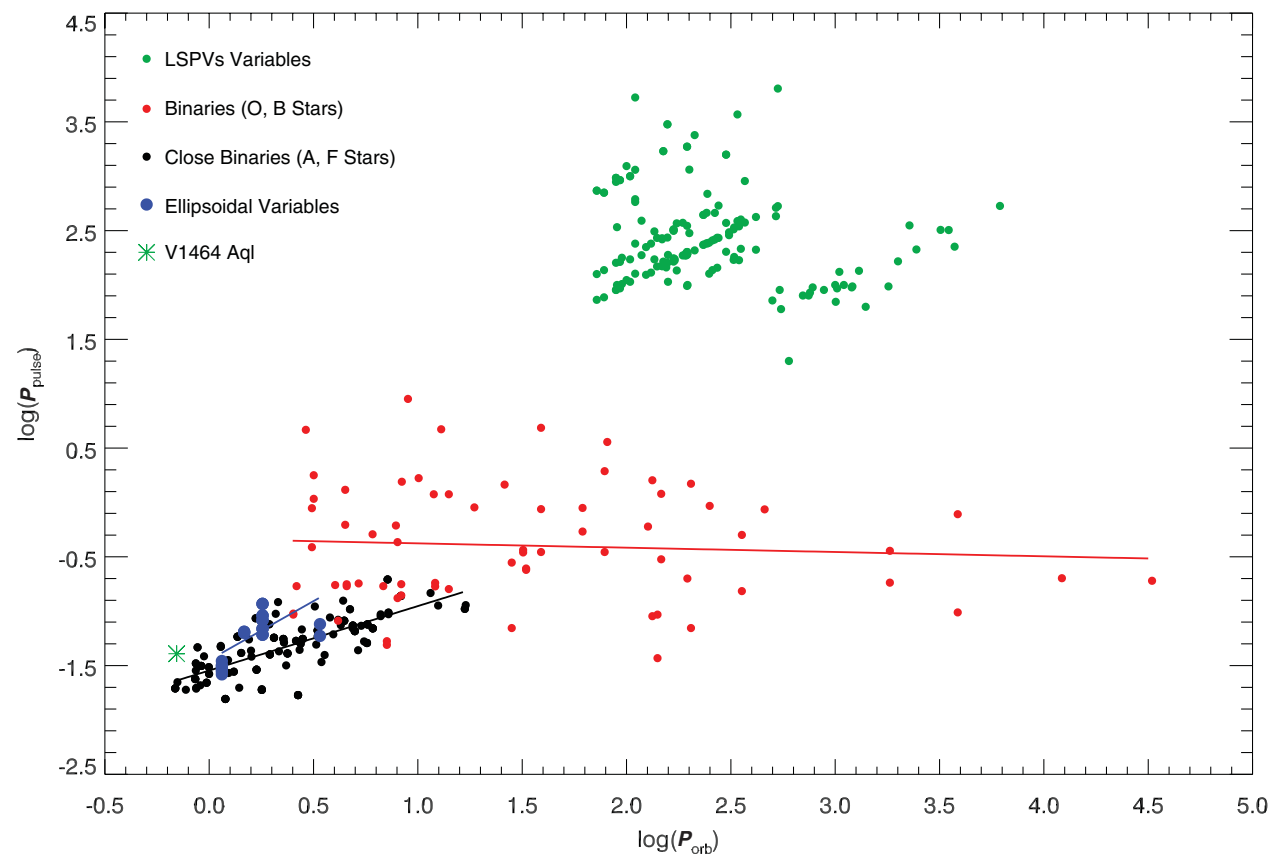

Figure 8. Location of V1464 Aql in the $\log \left(P_{\text {orb }}\right)-\log \left(P_{\text {pulse }}\right)$ plane among different types of binary stars. Filled circles represent the observed values, while the lines represent the theoretical linear fit of each group.

The fractional radii were found to be $r_{1}=0.441 \pm 0.002$ for the primary component and $r_{2}=0.378 \pm 0.002$ for the secondary component. In this case, the sum of fractional radii was computed as $r_{1}+r_{2} \simeq 0.80$. Thus, V1464 Aql seems to be in agreement with Kopal (1956)'s criteria for overcontact systems. The period analysis indicates the orbital period as $0.697822 \mathrm{~d}$. In addition, the temperature of the pri- mary component is $7420 \pm 192 \mathrm{~K}$ and that of the secondary component is $6232 \pm 161 \mathrm{~K}$. Although some contact binaries have components with some different surface temperature, they generally have the same surface temperature. Here, the primary component of V1464 Aql is hotter than the secondary component. Considering some characteristics of the system such as the short orbital period, small 
mass ratio, and hotter primary component, V1464 Aql seems to be in agreement with those of A-type W UMa binaries (Berdyugina 2005; Rucinski 1985). The period analyses reveal that the period of the short-term variation was found to be $58.482 \pm 0.002,58.482 \pm 0.001,60.966 \pm 0.002$, and $60.964 \pm 0.003$ min in $B V R I$ bands, respectively. However, we did not find any secondary frequency for the short-term variation. The period differences between each band may be due to the different sensitivity of each band. The sensitivity decreases from the $B$ to $I$ bands, because the amplitude of the pulsation is decreasing from the $B$ to $I$ bands. One can see from the standard deviations given in Table 4 and light curves in Figure 6, the scattering in the light curves increases from the $B$ to $I$ bands. Our period analysis methods depend on the statistical method. The analyses gave the best period statistically for the pulsation in each band. In this case, the most reliable periods are found from the $B$ and $V$ bands.

Figure 5 indicates that the short-term variation is dominant in shorter wavelengths rather than longer wavelengths. In fact, this is also seen clearly in Figure 6. The amplitude of the short-term variation gets larger from the $I$ band to the $B$ band. Moreover, the primary component is located in the Instability Strip on the main sequence, as seen in Figure 7. Considering the mass of the primary component and also the period of this short-term variation, this second variation should be caused due to the pulsation of the primary component. According to Aerts et al. (2010), the periods of $\delta$ Scuti stars are in the range of $18 \mathrm{~min}$ to $8 \mathrm{~h}$. Moreover, their mass is in the range of $1.50-2.50 \mathrm{M}_{\odot}$. The mass value of the component and the period of the short-term variation are in agreement with these values. In this case, this variation should be caused by the pulsation of a $\delta$ Scuti star.

As shown in Figure 1, the light curves seem to be scattered. There are also a few light curves in the ASAS database (Pojmánski 1997), which are also very scattered. However, the models of pulsation shown in Figure 6 demonstrate that the scattering in the light curves is caused due to the pulsation.

In the literature, there are four systems whose light curves have the same properties. In addition, these are ellipsoidal variables, which have a $\delta$ Scuti component. Aerts et al. (2002) found more than 10 frequencies due to the $\delta$ Scuti component in XX Pyx, whose orbital period is $1.15 \mathrm{~d}$. HD 207651 is a triple system. Henry et al. (2004) demonstrated that its component $\mathrm{A}$ is an ellipsoidal binary with a $\delta$ Scuti component. They found a few pulsation frequencies and the more possible one is $0.7354 \mathrm{~d}$. Chapellier et al. (2004) found $\sim 0.1169 \mathrm{~d}$ for the pulsation in the case of HD 173977. According to the light-curve analysis of Fekel \& Henry (2006), HD 149420 should be an eclipsing binary. However, it is likely to be an ellipsoidal variable. They found the pulsating periods of $\delta$ Scuti component as $0.076082 \mathrm{~d}$ and $0.059256 \mathrm{~d}$. In the case of V1464 Aql, we found the pulsating period as $\sim 0.040641$ d.

On the contrary, in the literature, the classification of V1464 Aql is a subject of debate (Pojmánski 1997; Duer- beck 1997; Pribulla \& Rucinski 2006; Pourbaix et al. 2004). Because of this, we plotted V1464 Aql among different binary types and variables in the $\log \left(P_{\text {orb }}\right)-\log \left(P_{\text {pulse }}\right)$ plane shown in Figure 8. As shown in the figure, V1464 Aql is indeed located near the close and ellipsoidal binaries with spectral type components A and F. This also supports the observation that the effective temperature found for the primary component of V1464 Aql is generally acceptable in the astrophysical sense. In addition, we also derived some new calibrations for different types of binaries. Both Turner (2011) and Liakos et al. (2012) have already derived the calibration for close binaries. In particular, Liakos et al. (2012) derived the calibrations separately for detached and semi-detached binaries. As given in Equation (3), combining all the close binaries listed by both Turner (2011) and Liakos et al. (2012), we derived the calibration together for close binaries from the spectral types A and F. Thus, we obtained more reliable calibrations, using a larger data set. In addition, the calibration has not been derived previously for binaries from the spectral types $\mathrm{O}$ and $\mathrm{B}$. Although one of their components is pulsating in these binaries, they are generally different from $\delta$ Scuti stars. As given in Equation (4), we tried, for the first time, to derive a similar calibration for these stars. A similar calibration was derived for ellipsoidal binaries, as given in Equation (5).

Rucinski \& Duerbeck (2006) noted that there are largely broadened lines that come from the visible component. They also found that the $v \sin i$ value of the component is $94 \pm 4$ $\mathrm{km} \mathrm{s}^{-1}$. Although this rapid rotation can cause the broadening in the lines, it can also be caused by pulsation. Moreover, the surface temperature of the primary component is $7420 \pm 192 \mathrm{~K}$, while it is $6232 \pm 161 \mathrm{~K}$ for the secondary component. In this case, it is expected that we see double lines in the spectrum, though the inclination of the system (i) is $38^{\circ} .45$. However, Rucinski \& Duerbeck (2006) stated that V1464 Aql is a single-lined system, and the amplitude of the radial velocity is $30.62 \pm 2.35 \mathrm{~km} \mathrm{~s}^{-1}$. The amplitude of the radial velocity is large enough that it can easily confuse one's mind. In this case, we also expected to see the lines of the secondary component and thus its radial velocity. On the contrary, considering both the spectral and photometrical results together, rapid rotation and pulsation nature of the primary component can have some effects on its spectrum in the case of a lower inclination $(i)$ at $38^{\circ} .45$. The broadening of the lines should make the lines coming from the secondary component disappear.

Consequently, V1464 Aql is a new candidate for the ellipsoidal system with a pulsating component. Future work will study its pulsating nature in more detail, thus revealing the absolute nature of the system.

\section{ACKNOWLEDGMENTS}

The author acknowledges the generous observing time awarded to the Ege University Observatory. The author also thanks the referee for useful comments that significantly improved the paper. 


\section{REFERENCES}

Aerts, C., Christensen-Dalsgaard, J., \& Kurtz, D. W. 2010, in Asteroseismology, ed. W. B. Burton \& V. Trimble (New York: Springer), 49

Aerts, C., Handler, G., Arentoft, T., Vandenbussche, B., Medupe, R., \& Sterken, C. 2002, MNRAS, 333, L35

Aerts, C., \& Harmanec, P. 2004, ASPC, 318, 325

Ammons, S. M., Robinson, S. E., Strader, J., Laughlin, G., Fischer, D., \& Wolf, A. 2006, ApJ, 638, 1004

Beech, M. 1985, Ap\&SS, 117, 69

Berdyugina, S. V. 2005, LRSP, 2,8

Chapellier, E., et al. 2004, A\&A, 426, 247

Cunha, M. S., et al. 2007, A\&AR, 14, 217

Derekas, A., Kiss, L. L., Bedding, T. R., Kjeldsen, H., Lah, P., \& Szabó, Gy. M. 2006, ApL, 650, 55

Duerbeck, H. W. 1997, IBVS, 4513, 1

Essam, A., Saad, S. M., Nouh, M. I., Dumitrescu, A., El-Khateeb, M. M., \& Haroon, A. 2010, NewA, 15, 227

Faigler, S., \& Mazeh, T. 2011, MNRAS, 415, 3921

Faigler, S., Mazeh, T., Quinn, S. N., Latham, D. W., \& Tal-Or, L. 2012, ApJ, 746, 185

Fekel, F. C., \& Henry, G. W. 2006, AJ, 131, 1724

Foster, G. 1995, AJ, 109, 1889

Girardi, L., Bressan, A., Bertelli, G., \& Chiosi, C. 2000, A\&AS, 141,371

Hardie, R. H. 1962, in Astronomical Techniques, ed. W. A. Hiltner (Chicago, IL: University of Chicago Press), 178

Henry, G. W., Fekel, F. C., \& Henry, S. M. 2004, AJ, 127, 1720

Kharchenko, N. V., Piskunov, A. E., Röser, S., Schilbach, E., Scholz, R.-D., \& Zinnecker, H. 2009, A\&A, 504, 681

Kim, S.-L., Lee, J. W., Lee, C.-U., \& Youn, J.-H. 2010, PASP, 122, 1311

Kiss, L. L., Szatmáry, K., Cadmus, R. R. Jr, \& Mattei, J. A. 1999, A\&A, 346, 542

Kopal, Z. 1956, AnAp, 19, 298

Kwee, K., \& van Woerden, H. 1956, BAN, 12, 464

Lampens, P. 2006, ASPC, 349, 153

Lenz, P., \& Breger, M. 2005, CoAst, 146, 53

Liakos, A., Niarchos, P., Soydugan, E., \& Zasche, P. 2012, MNRAS, 422,1250

Lucy, L. B. 1967, ZA, 65, 89
Martin, B. E., Hube, D. P., \& Brown, C. 1991, PASP, 103, 424

Martin, B. E., Hube, D. P., \& Lyder, D. A. 1990, PASP, 102, 1153

Motulsky, H. 2007, in GraphPad Prism 5: Statistics Guide (San Diego, CA: GraphPad Software Inc. Press), 94

Morris, S. L. 1985, ApJ, 295, 143

Morris, S. L., \& Naftilan, S. A. 1993, ApJ, 419, 344

Nie, J. D., Zhang, X. B., \& Jiang, B. W. 2010, AJ, 139, 1909

Olivier, E. A., \& Wood, P. R. 2003, ApJ, 584, 1035

Pigulski, A. 2006, ASPC, 349, 137

Pojmánski, G. 1997, AcA, 47, 467

Pourbaix, D., et al. 2004, A\&A, 424, 727

Pribulla, T., \& Rucinski, S. M. 2006, AJ, 131, 2986

Prša, A., \& Zwitter, T. 2005, ApJ, 628, 426

Rolland, A., Costa, V., Rodriguez, E., Amado, P. J., GarciaPelayo, J. M. Lopez de Coca, P., \& Olivares, I. 2002, CoAst, 142,57

Rucinski, S. 1985, in Interacting Binary Stars, ed. J. E. Pringle \& R. A. Wade (Cambridge: Cambridge University Press), 1

Rucinski, S. M. 1969, AcA, 19, 245

Rucinski, S. M., \& Duerbeck, H. W. 2006, AJ, 132, 1539

Scargle, J. D. 1982, ApJ, 263, 835

Soydugan, E., Soydugan, F., Demircan, O., \& Ibanoğlu, C. 2006, MNRAS, 370, 2013

Stellingwerf, R. F. 1978, ApJ, 224, 953

Tokunaga, A. T. 2000, in Allen's Astrophysical Quantities, ed. A. N. Cox (4th ed.; New York: Springer), 143

Turner, G. H. 2011, The Connection between Delta Scuti Stars and Close Binary Parameters Thesis, Ball State University, Muncie van Hamme, W. 1993, AJ, 106, 2096

Wall, J. W., \& Jenkins, C. R. 2003, in Practical Statistics for Astronomers (Cambridge: Cambridge University Press), 79

Wilson, R. E. 1990, ApJ, 356, 613

Wilson, R. E., \& Devinney, E. J. 1971, ApJ, 166, 605

Winkler, L. 1967, AJ, 72, 226

Wright, C. O., Egan, M. P., Kraemer, K. E., \& Price, S. D. 2003, AJ, 125, 359

Yang, Y.-G. 2010, Ap\&SS, 326, 125

Yang, Y.-G., Wei, J.-Y., \& He, J.-J. 2008, AJ, 136, 594

Zacharias, N., Monet, D. G., Levine, S. E., Urban, S. E., Gaume, R., \& Wycoff, G. L. 2005, yCat., 1297, 0 (originally published in 2004, AAS, 205, 4815) 